\title{
The Direction of Evolution in the Drosophila melanogaster Species Subgroup Based on Functional Analysis of the Crystal Cells
}

\author{
T. M. RIZKI AND ROSE M. RIZKI \\ Department of Zoology, Division of Biological Sciences, The University of Michigan, Ann \\ Arbor, Michigan 48109
}

\begin{abstract}
The crystal cells in the hemolymph of Drosophila melanogaster contain paracrystalline inclusions and phenol oxidase. Melanization of this type of blood cell can be induced by treatment of larvae with hot water. By using this functional criterion to establish cell homology we compared the shape of the inclusions in the crystal cells of the $D$. melanogaster species subgroup and a number of distantly related species of Drosophila. The crystal cell inclusions in four of the sibling species (simulans, erecta, mauritiana, yakuba) are the same as those found in $D$. melanogaster. In the fifth species, $D$. teissieri, the crystal cell inclusions resemble those found in the hemocytes of the other larval drosophilids that were examined rather than those of $D$. melanogaster. On the basis of these observations and the phylogenetic relationships deduced from analysis of chromosomal inversions by Lemeunier and Ashburner ('76), we propose that $D$. teissieri is closer to the ancestral form or is the ancestral species of the melanogaster species subgroup of Drosophila. This is the first instance in which a hemocyte type has been used to determine the direction of evolution within a subgroup of insect species.
\end{abstract}

The larval hemolymph of Drosophila melanogaster contains two classes of hemocytes, plasmatocytes and crystal cells (Rizki, '57a). Plasmatocytes differentiate into podocyte and lamellocyte variants but the crystal cells, comprising $5-10 \%$ of the larval hemocyte population, remain uniquely distinguishable by their prominent paracrystalline inclusions. Such cytoplasmic structures have not, to our knowledge, been reported in the blood cells of other insects or even other species of Drosophila. Yet the function ascribed to these cells, storage of the components involved in hemolymph phenol oxidase activity (Rizki and Riz$\mathrm{ki}$, '59), is a common attribute of insect hemolymph.

Studies on a recently isolated mutant gene, Black cells (Bc, 2-80.6 $)$, agree with the localization of hemolymph phenol oxidase activity to the crystal cells (Rizki and Rizki, '79a; Rizki et al., submitted for publication). Blood cells with paracrystalline inclusions are absent in $B c$ larvae that contain instead melanized cells in the hemolymph and lymph glands, suggesting that the blackened cells are a mutant form of the crystal cells. When the cells in this mutant are melanized, the phenol oxidase within them is destroyed; hence $B c$ larvae lack phenol oxidase activity. Furthermore, melanization of the crystal cells in $\mathrm{Bc}^{+}$larvae can be induced by hot water treatment, and the distribution of heat-induced black cells in these larvae mimics the distribution of black cells in $B c$ mutant larvae. From this series of observations it is clear that two methods can be used to identify crystal cells in $D$. melanogaster: structurally, on the basis of their paracrystalline inclusions; and functionally, by hot water treatment to blacken them. Utilizing these criteria we examined the hemocytes of a number of sibling species of $D$. melanogaster and some other members of the family Drosophilidae. For the latter group, no effort was made to be exhaustive; we used species that were available in our laboratory. The five sibling species of $D$. melanogaster used are those studied by Lemeunier and Ashburner ('76). The present report summarizes these findings on Drosophila hemocytes and suggests that analysis of hemocyte types may be useful in delineating phylogenetic relationships in Drosophila. 


\section{MATERIALS AND METHODS}

The sibling species used for this study were: $D$. melanogaster (Ore- $R$ wild type), $D$. erecta (154.1), D. simulans (C135.20), D. yakuba (115), D. teissieri (128.2), and D. mauritiana. The other species examined were: $D$. pseudoobscura, $D$. virilis, $D$. novamexicana, $D$. willistoni, D. nebulosa, D. buskii, and Zaprionus vittiger.

All larvae were grown on cream of wheat medium with live yeast at $18^{\circ} \mathrm{C}$ except $Z a$ prionus larvae, which were grown on corn meal-agar medium and $D$. buskii larvae, which were grown on medium containing flax seed. Mid-third instar larvae were washed with $1.2 \% \mathrm{NaClO}$ and rinsed well with distilled $\mathrm{H}_{2} \mathrm{O}$ to remove extraneous food particles from the body surface. Larvae were opened by tearing the body wall, taking care not to rupture internal organs, and allowing the hemolymph to flow into a small drop of buffered formaldehyde $(2.3 \%$ in phosphate buffer at $\mathrm{pH}$ 7.2). After 4 minutes dilute Giemsa stain was added to the hemolymph sample, and the stained cells were examined. At least five camera lucida drawings of crystal cells were made for each species to assure that variations in shape and size of the inclusions were recorded.

To establish the functional identity of the cells with inclusions, larvae were immersed in hot water at $70^{\circ} \mathrm{C}$. When blackening of hemocytes became apparent, the treated larvae were examined either directly under transmitted light (compound microscope: $12.5 \times 16$ or $12.5 \times 25$ magnification), or the larvae were opened in Carnoy fixative and tissues with attached darkened cells were processed and examined. The cytoplasmic inclusions are still visible in the cells that are only lightly tanned, so the cells were not permitted to blacken fully. The inclusions in the melanizing cells and in the stained preparations were compared.

\section{RESULTS}

The cytoplasmic inclusions in the crystal cells of $D$. melanogaster have a strikingly regular shape. The two longer sides of the structures appear parallel with ends that are either rectangular or conical. Since photographs of $D$. melanogaster crystal cells have been published previously (Rizki and Rizki, '59), none are included here. However, the paracrystalline inclusions in four of the sibling species, $D$. simulans, $D$. mauritiana, $D$. $y a k u b a$, and $D$. erecta are indistinguishable from those found in D. melanogaster crystal, cells, and representative crystal cells from these four species are included (Fig. 1). There are variations in size of the paracrystalline inclusions, and the number of inclusions per cell also differs among crystal cells taken from the same hemolymph sample. Cells similar to the four cells in this plate, each taken from a different sibling species, can all be found in a single sample taken from any one of the sibling species, including $D$. melanogaster. Treatment with hot water blackened the cells with crystals in the four sibling species.

Figure 2 contains photographs of crystal cells from $D$. teissieri, another sibling species of $D$. melanogaster. The inclusions in these cells lack the general rectangular appearance found in the crystal cells of the other five species of the melanogaster subgroup of Drosophila. They appear chunky and globular The inclusions are quantitatively variable but none of the inclusions resemble those found in the other sibling species. That these cells are functionally similar to the crystal cells of the other melanogaster sibling species can be demonstrated by immersion of the larvae in hot water, for they respond to this treatment precisely as do the crystal cells.

In all the larvae from outside the melanogaster species subgroup the hemocytes with inclusions resembled those found in $D$. teissieri rather than those found in $D$. melanogaster (Fig. 3). There were size and quantitative variations, but none similar to the melanogaster type were found. For each species the functional relationship to the melanogaster crystal cell was established by hot water treatment.

\section{DISCUSSION}

This report is the first attempt to use a functional criterion for classification of a specific hemocyte type in the genus Drosophila. Classification of insect hemocytes relies primarily on morphological characteristics, so categorization of cells to a common class among different insects is possible only when there are distinctly comparable structural traits. To draw analogies in the absence of such similarity or in the absence of information on the functions of a given cell type is unwise, since hemocytes display morphological variations in response to physiological changes in the hemolymph; they also show variations during their own stages of development (Rizki, '78). D. melanogaster with its many mutant strains has been exceptionally useful for studying hemocyte interrelation- 

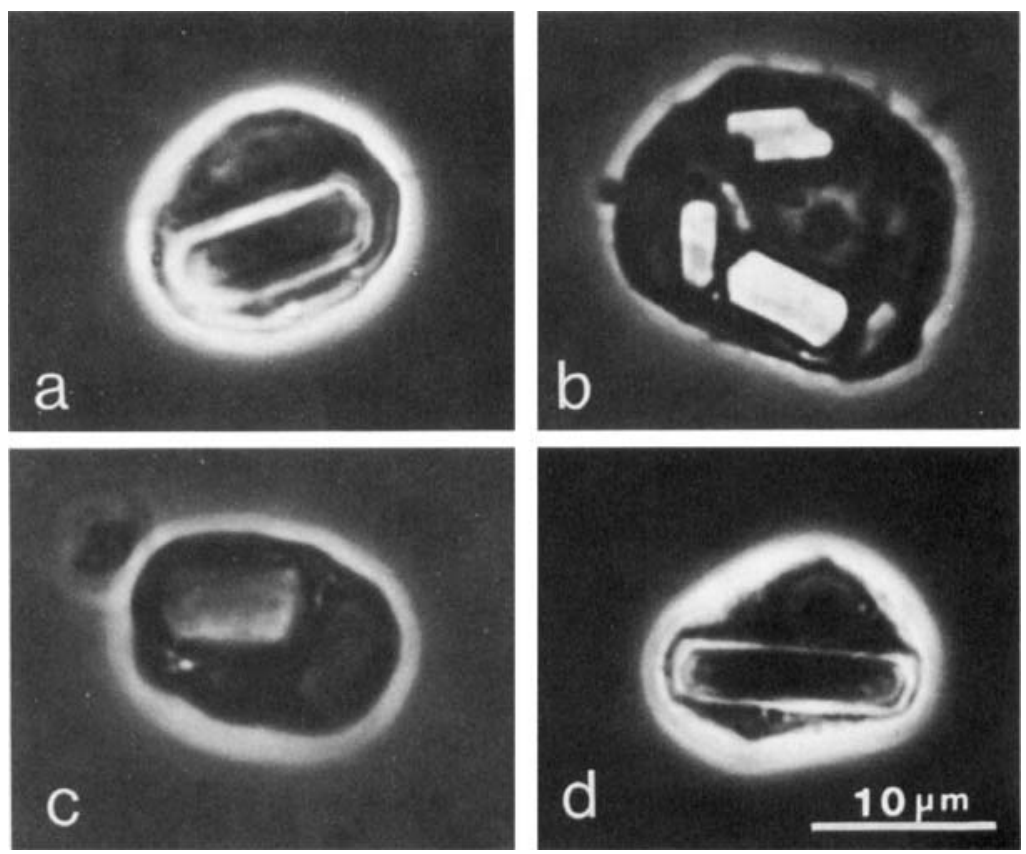

Fig. 1. Morphology of the paracrystalline inclusions in the crystal cells of: $a, D$. simulans; $b, D$. mauritiana; c, D. yakuba; d, D. erecta.
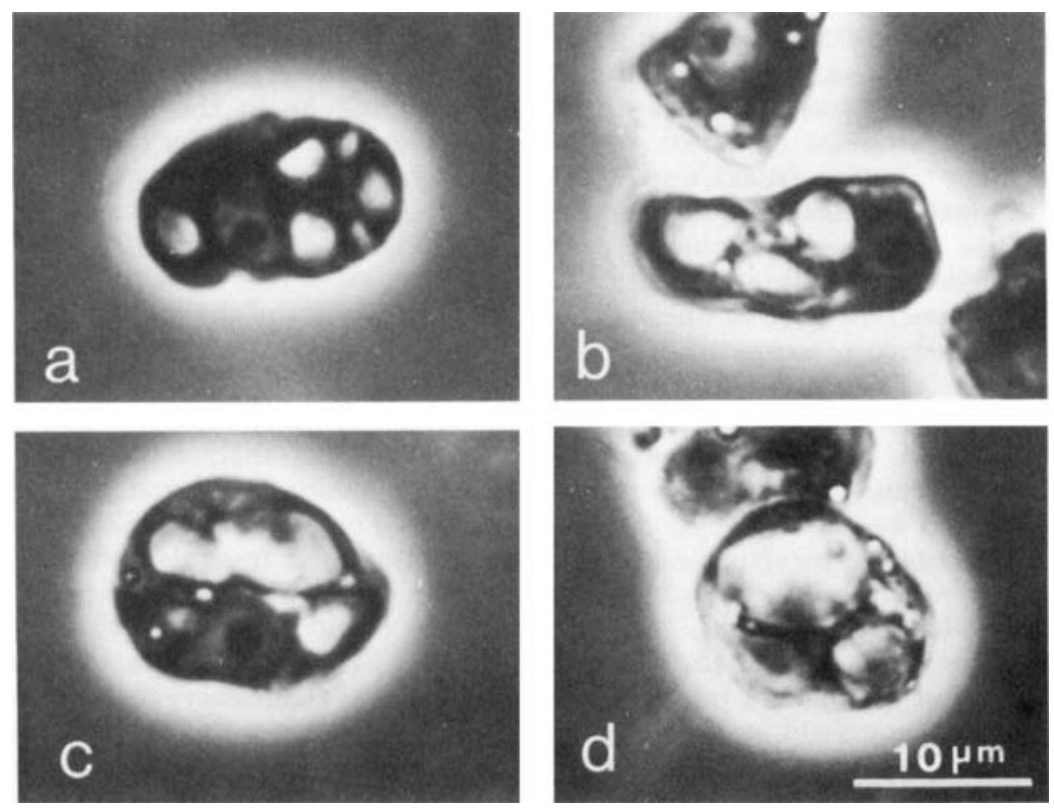

Fig. 2. Crystal cells of D. teissieri. 

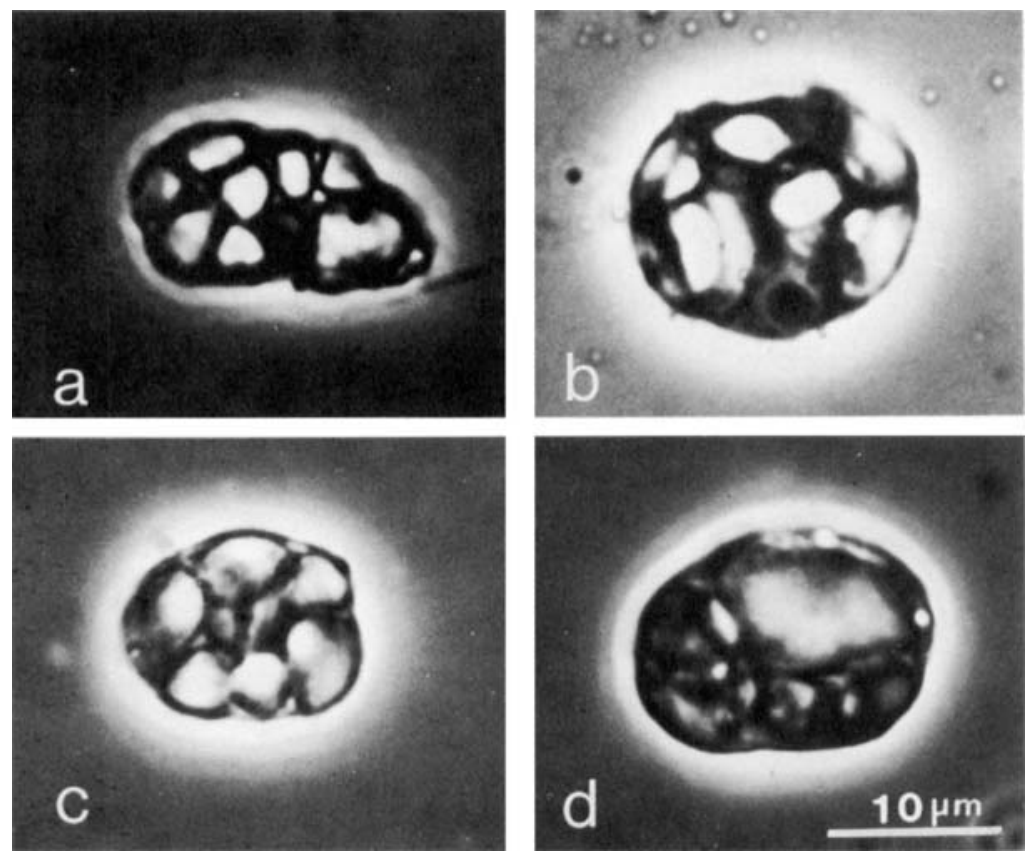

Fig. 3. Crystal cells of: a, D. willistoni; b, D. pseudoobscura; c, D. novamexicana; d, Zaprionus vittiger.

ships: melanotic tumor strains provided the material for studying the plasmatocytes and their morphological variants (Rizki, '57b; Rizki and Rizki, '79b); the $B c$ mutant confirmed the crystal cell as the source of hemolymph phenol oxidase activity and lead to a simple technique for releasing melanization in the crystal cells of $\mathrm{Bc}^{+}$larvae (Rizki and Rizki, 79a). With the latter method hemocytes that are not otherwise readily comparable by morphological criteria can be grouped into one category, and we are now able to unravel the enigma in the literature concerning crystal cells in drosophilids. Yeager's ('45) terminology was adopted for classification of the blood cells in D. willistoni (Rizki, '53) with the exception that one category of cells was designated crystalloid cells. These hemocytes contain highly refractile, birefringent rodlets and are found in young larvae. In subsequent studies on $D$. melanogaster (Rizki, '57a) the term crystal cell was adopted for the cell type that is the subject of the present report. Crystal cells are found throughout larval life in $D$. melanogaster and are morphologically distinct from crystalloid cells. On the basis of our published work, Nappi ('70) concluded that "crystalloid cells of $D$. willistoni and the crystal cell of $D$. melanogaster are unquestionably morphologically similar" although Rizki and Rizki ('59) did not "analogize the crystalloid cells of $D$. willistoni" with the crystal cells in $D$. melanogaster. From the present study it is clear that the crystal cells in $D$. melanogaster are analogous to the cells in $D$. willistoni described as spheroidocytes (Rizki, '53); there is as yet no information suggesting a relationship, if any, between the crystalloid cells of $D$. willistoni and the crystal cells of $D$. melanogaster.

The fragility of the crystal cells is a distinctive feature of this hemocyte type in $D$. melanogaster (Rizki, '57a). When the body wall is opened or hemolymph is removed from the hemocoel, the inclusions within these cells begin to disintegrate and the cells often rupture. We proposed that effective separation of phenol oxidase from its substrate is achieved within the crystal cell by storage of the latter in the paracrystalline inclusions (Rizki and Rizki, '59). Disturbances that influence the integrity of the paracrystalline inclusions will allow contact of substrate with enzyme, thus resulting in melanization. Since the crystal cell inclusions are unusually sensitive to experimental manipulations, we cannot ignore the possibility that the morphological difference observed between the melanogaster and 
non-melanogaster cell types is due to factors in the cells or hemolymph that influence the stability of the inclusions, rather than a difference in the structural components of the inclusions themselves. Hopefully, biochemical and/or ultrastructural examinations of these cell types will answer this question. In the meantime, the distinction between these two groups is apparent by utilizing the fixation methods employed in the present study.

A brief summary of the species included in the melanogaster subgroup of Drosophila is presented by Lemeunier and Ashburner ('76). These authors analyzed the banding patterns of the salivary gland chromosomes of the six sibling species, and on the basis of the overlapping inversions established phylogenetic relationships among the species. Their scheme includes bidirectional arrows for, as stressed by these authors, chromosomal inversion phylogenies are seldom useful for determining the progression of evolution from primitive to more recent species. Additional distinguishing features must be sought, and these might then be combined with the information from inversion phylogenies to delineate lines of descent. Among the morphological traits generally useful for distinguishing sibling species are the structural features of the male genitalia. Interestingly, structural similarity of male genitalia does not necessarily indicate genetic closeness, for the male genitalia of the sibling species $D$. pseudoobscura and $D$. persimilis are distinguishable with difficulty (Rizki, '51) whereas those of $D$. simulans and $D$. melanogaster are quite distinct (Sturtevant, '29). Indeed, the male genitalia of the six sibling species of the melanogaster subgroup are sufficiently different to allow recognition of these species (Bock and Wheeler, '72; Tsacas and David, '74; Tsacas and Lachaise, '74), but there do not appear to be differences and similarities in these organs that would be useful for analyzing the direction of evolution. A recent analysis of polypyrimidine stretches in drosophilids including sibling species in the melanogaster subgroup shows a similarity of sequences in melanogaster, mauritiana, and simulans DNA, but erecta, yakuba, and teissieri do not have polypyrimidine sequences that will hybridize with $D$. melanogaster polypyrimidines (Cseko et al., '79). Therefore, evolutionary directions for this subgroup cannot be assessed by these methods. We believe that the unique morphology of the inclusions in the crystal cells can be used for this purpose. Five of the six sibling species share a similar morphology of these structures whereas the remaining species, $D$. teissieri, differs from these and resembles the other Drosophila species that we examined.

We stress that the sampling of species outside the melanogaster subgroup examined in this study is very limited, and we do not imply that the melanogaster-type crystal cell occurs only in this subgroup. An extensive review of the drosophilids and their phylogenetic interrelationships is presented by Throckmorton (75). Aside from the melanogaster subgroup, we examined the hemocytes of willistoni, nebulosa, and pseudoobscura, representing at least two distantly related species groups of Sophophorans, two species from the virilis group, a species of the genus Zaprionus, and $D$. buskii in the subgenus Dorsilopha. The inclusions in the hemocytes of all these species are like those of $D$. teissieri. If we accept that the widely occurring characteristic is the ancestral trait, then $D$. teissieri is closer to the ancestral form or is the ancestral species of the melanogaster species subgroup. This suggestion is combined with the scheme of Lemeunier and Ashburner ('76) in Figure 4. Such an evolutionary pattern supports the conclusion of Tsacas and Lachaise ('74) and Throckmorton ('75) that the melanogaster spe-

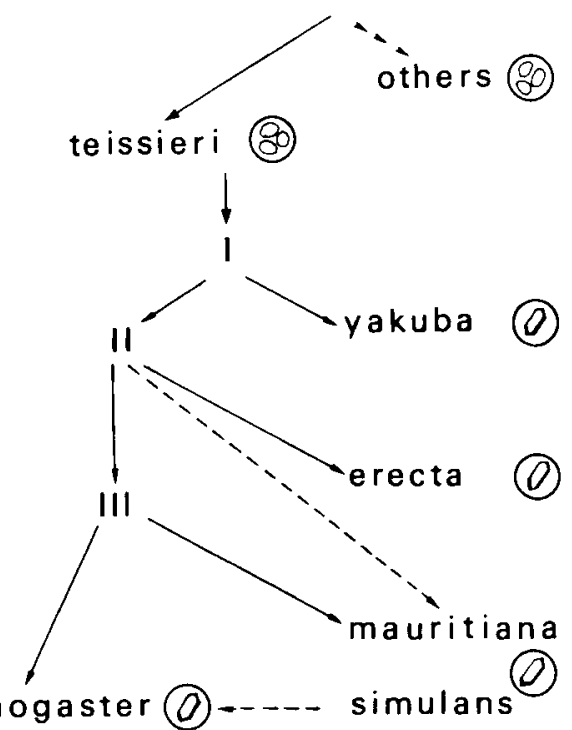

Fig. 4. The proposed direction of evolution in the $D$. melanogaster species subgroup deduced from analysis of morphology of the inclusions in the crystal cells. This scheme is based on the phylogeny of inversions presented by Lemeunier and Ashburner (76); I, II, and III are hypothetical inversion steps and the dotted lines represent an alternate scheme proposed by these authors. 
cies subgroup arose in Africa since $D$. teissieri is endemic to Africa (Tsacas, '71). Furthermore, D. yakuba and D. erecta are also African endemic species; $D$. mauritiana is known from the island of Mauritius (Tsacas and David, '74).

Mating preference has been suggested as a means for determining phylogenetic relationships among Drosophila species by assuming that females of an ancestral species discriminate against males of a derived species (Kaneshiro, '76). The success of reciprocal hybrid matings between mauritiana, simulans, and melanogaster was measured by Watanabe and Kawanishi ('79). These authors, however, suggest that mating preference is exerted by the females of the newly derived species and on the basis of this assumption propose the evolutionary scheme from melanogaster $\rightarrow$ simulans $\rightarrow$ mauritiana. If Kaneshiro's ('76) assumption is accepted, then the same data predict the direction of evolution as mauritiana $\rightarrow$ melanogaster. Crystal cell morphology together with inversion phylogeny suggests that $D$. melanogaster is the more recently derived species of this group. In line with Throckmorton's (75) reasoning on the wide spread distribution of $D$. melanogaster and its association with man, it is interesting to consider that this association was first established in Africa.

\section{ACKNOWLEDGMENTS}

We are especially grateful to Dr. R. C. Woodruff of Bowling Green State University for providing us with the sibling species of $D$. melanogaster and to Dr. L. H. Throckmorton of The University of Chicago for helpful comments on the manuscript. This research was supported by Grant No. CA-16619 awarded by the National Cancer Institute, DHEW.

\section{LITERATURE CITED}

Bock, I.R., and M.R. Wheeler (1972) The Drosophila melanogaster species group. Stud. Genet. VII. University of Texas Publications, 7213: 1-102.
Cseko, Y.M.T., N.A. Dower, P. Minoo, L. Lowenstein, G.R. Smith, J. Stone, and R. Sederoff (1979) Evolution of polypyrimidines in Drosophila. Genetics, 92:459-484.

Kaneshiro, K.Y. (1976) Ethological isolation and phylogeny in the planitibia subgroup of Hawaiian Drosophila. Evolution, 30:740-745.

Lemeunier, F., and M. Ashburner (1976) Relationships within the melanogaster species subgroup of the genus Drosophila (Sophophora). II. Phylogenetic relationships between six species based upon polytene chromosome banding sequences. Proc. R. Soc. Lond. B, 193:275-294.

Nappi, A.J. (1970) Hemocytes of larvae of Drosophila euronotus (Diptera: Drosophilidae). Ann. Entomol. Soc. Am., 63:1217-1224.

Rizki, T.M. (1951) Morphological differences between two sibling species, Drosophila pseudoobscura and Drosophila persimilis. Proc. Natl. Acad. Sci., 37: 156-159.

Rizki, T.M. (1953) The larval blood cells of Drosophila willistoni. J. Exp. Zool., 123:397-411.

Rizki, T.M. (1957a) Alterations in the haemocyte population of Drasophila melanagaster. J. Morphol., 100:437-458.

Rizki, T.M. (1957b) Tumor formation in relation to metamorphosis in Drosophila melanogaster. J. Morphol., 100:459-472.

Rizki, T.M. (1978) The circulatory system and associated cells and tissues. In: The Genetics and Biology of Drosophila. M. Ashburner and T.R.F. Wright, eds. Academic Press, London, Vol. 2b, pp. 397-452.

Rizki, T.M., and R.M. Rizki (1959) Functional significance of the crystal cells in the larva of Drosophila melanogaster. J. Biophys. Biochem. Cytol., 5:235-240.

Rizki, R.M., and T.M. Rizki (1979a) Phenogenetics of Black cells (Bc). Genetics, 91:s103-104.

Rizki, R.M., and T.M. Rizki (1979b) Cell interactions in the differentiation of a melanotic tumor in Drosophila. Differentiation, 12:167-178.

Sturtevant, A.H. (1929) The genetics of Drasophila simulans. Carnegie Inst. Wash., 339:1-62.

Throckmorton, L.H. (1975) The phylogeny, ecology, and geography of Drosophila. In: Handbook of Genetics, Vol. 3. R.C. King, ed. Plenum Press, New York, pp. 421-469.

Tsacas, L. (1971) Drosophila teissieri, nouvelle espèce africaine du groupe melanogaster et note sur deux autres espèces nouvelles pour l'Afrique (Diptera: Drosophilidae). Bull. Soc. Ento. France, 76:35-45.

Tsacas, L., and J. David (1974) Drosophila mauritiana n. sp. du groupe melanogaster de l'Ile Maurice (Dipt.: Drosophilidae). Bull. Soc. Ento. France, 79:42-46.

Tsacas, L., and D. Lachaise (1974) Quatre nouvelles espèces de la Cote d'Tvoire du genre Drosophila, groupe melanogaster, et discussion de l'origine du sous-groupe melanogaster (Diptera: Drosophilidae). Ann. Univ. Abidjan, E7(1): 193-211.

Watanabe, T.K., and M. Kawanishi (1979) Mating preference and the direction of evolution in Drosophila. Science, 205: 906-907.

Yeager, J.F. (1945) The blood picture of the southern armyworm (Prodenia eridania). J. Agric. Res., 71:1-40. 